\title{
Is nuclear matter perturbative with low-momentum interactions?
}

\author{
S.K. Bogner ${ }^{1}$, A. Schwenk ${ }^{2}$, R.J. Furnstahl ${ }^{1}$ and A. Nogga ${ }^{3}$ \\ ${ }^{1}$ Department of Physics, The Ohio State University, Columbus, OH 43210 \\ ${ }^{2}$ Nuclear Theory Center, Indiana University, Bloomington, IN 47408 \\ ${ }^{3}$ Institut für Kernphysik, Forschungszentrum Jülich, 52425 Jülich, Germany
}

\begin{abstract}
The nonperturbative nature of inter-nucleon interactions is explored by varying the momentum cutoff of a two-nucleon potential. Conventional force models, which have large cutoffs, are nonperturbative because of strong short-range repulsion, the iterated tensor interaction, and the presence of bound or nearly-bound states. But for low-momentum interactions with cutoffs around $2 \mathrm{fm}^{-1}$, the softened potential combined with Pauli blocking leads to corrections in nuclear matter in the particleparticle channel that are well converged at second order in the potential, suggesting that perturbation theory can be used in place of Brueckner resummations. Calculations of nuclear matter using the low-momentum two-nucleon force $V_{\text {low }} k$ with a corresponding leading-order three-nucleon $(3 \mathrm{~N})$ force from chiral effective field theory (EFT) exhibit nuclear binding in the Hartree-Fock approximation, and become less cutoff dependent with the inclusion of the dominant second-order contributions. The role of the $3 \mathrm{~N}$ force is essential to obtain saturation, and the contribution to the total potential energy is compatible with EFT power-counting estimates.
\end{abstract}

\section{Introduction}

Conventional wisdom among nuclear physicists, as summarized by Bethe in his review of over 30 years ago [1], holds that successful nuclear matter calculations must be nonperturbative in the inter-nucleon interactions. The possibility of a soft potential providing a perturbative solution to the nuclear matter problem was discarded at that time, and saturation firmly identified

Email addresses: bogner@mps.ohio-state.edu (S.K. Bogner), schwenk@indiana.edu (A. Schwenk), furnstahl.1@osu.edu (R.J. Furnstahl), a.nogga@fz-juelich.de (A. Nogga).

Preprint submitted to Elsevier Science 6 February 2008 
with the density dependence due to the tensor force [1]. Subsequent work on the nuclear matter problem [2] has not significantly altered the general perspective or conclusions of Bethe's review (although the role of three-nucleon (3N) forces has been increasingly emphasized). However, recent results from renormalization-group-based low-momentum potentials, coupled with insight from effective field theory (EFT), mandate that these conclusions be revisited. These developments affect not only the technical conclusions but also the underlying philosophy of nuclear matter calculations.

Nonperturbative behavior in the particle-particle channel for nuclear forces arises from several sources. First is a strongly repulsive short-range interaction, which requires at least a summation of particle-particle ladder diagrams [1]. Second is the tensor force, e.g., from pion exchange, which is highly singular at short distances, and requires iteration in the triplet channels [3,4]. Third is the presence of low-energy bound states or nearly-bound states, which are found in the nucleon-nucleon (NN) S-waves. These states imply poles in the scattering $T$ matrix that render the perturbative Born series divergent. All of these nonperturbative features are present in conventional high-precision NN potentials, such as the Argonne $v_{18}$ potential [5], which has been used in the most accurate $a b$ initio calculations of nuclei and nuclear matter to date $[6,7,8]$.

The philosophy behind the standard approach to nuclear matter is to attack these features head-on. This attitude was succinctly stated by Bethe [1]:

"The theory must be such that it can deal with any NN force, including hard or 'soft' core, tensor forces, and other complications. It ought not to be necessary to tailor the $\mathrm{NN}$ force for the sake of making the computation of nuclear matter (or finite nuclei) easier, but the force should be chosen on the basis of NN experiments (and possibly subsidiary experimental evidence, like the binding energy of $\left.\mathrm{H}^{3}\right)$."

In contrast, the EFT perspective stresses that the potential is not an observable to be fixed from experiment (there is no "true potential"), but that an infinite number of potentials are capable of accurately describing low-energy physics [9]. In order to be predictive and systematic, an organization ("power counting") must be present to permit a finite truncation of possible terms in the potential. If a complete inter-nucleon potential is used, including manynucleon interactions, then all observable results should be equivalent up to truncation errors. The EFT philosophy applied to nuclear matter implies using this freedom to pick a convenient and efficient potential under the conditions of interest.

A particularly useful class of energy-independent NN potentials is characterized by a momentum cutoff $\Lambda$, which limits the resolution of details in the 
potential. One starts with an accurate NN potential (such as Argonne $v_{18}$ [5] or a chiral $\mathrm{N}^{3} \mathrm{LO}$ potential $[10,11]$ ) and a sufficiently high "bare" cutoff (all potentials are cut off at high momentum by form factors or other regulators) and evolves the cutoff to lower values, while requiring that observables (e.g., phase shifts) for external momenta up to the cutoff are unchanged $[12,13]$. This can be accomplished by renormalization-group ( $\mathrm{RG}$ ) equations that preserve the half-on-shell $T$ matrix elements (and therefore the physical on-shell amplitude) or, equivalently, by Lee-Suzuki transformations [12,13,14] (effective interactions in momentum space have also been derived in Ref. [15]). Varying the cutoff can be used as a powerful tool to study the underlying physics scales, to evaluate the completeness of approximate calculations, and to estimate truncation errors from omitted higher-order contributions.

These variable-cutoff potentials reveal the resolution or scale dependence of the first two sources of nonperturbative behavior, which are dampened as high-momentum intermediate states are eliminated. In free space, the third source of nonperturbative behavior remains independent of the cutoff because the pole positions of shallow bound states that necessitate fine tuning are physical observables. However, this fine tuning is eliminated in the medium at sufficiently high density. Our results show that a low-momentum two-nucleon

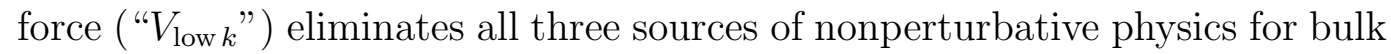
properties of nuclear matter. In short, a repulsive core is not constrained by phase shifts and is essentially removed by even a moderately low-momentum cutoff, the tensor force is tamed by a sufficiently low cutoff, and the shallow bound states become perturbative as a result of Pauli blocking. For cutoffs around $2 \mathrm{fm}^{-1}$, which preserve phase shifts up to $330 \mathrm{MeV}$ laboratory energy, the Born series in nuclear matter is well converged at second order in the potential, suggesting that perturbation theory can be used in place of Brueckner resummations.

While evolving a soft potential from higher momentum is a new development in nuclear physics $[12,13]$, attempts to use soft potentials for nuclear matter were common in the 1960's and early 1970's [16]. It had long been observed that a strongly repulsive core is not resolved until eight times nuclear saturation density [1]. Thus, saturation is not driven by a hard core (unlike liquid ${ }^{3} \mathrm{He}$ ). However, these soft potentials were abandoned because they seemed incapable of quantitatively reproducing nuclear matter properties. Their requiem was given by Bethe [1]:

"Very soft potentials must be excluded because they do not give saturation; they give too much binding and too high density. In particular, a substantial tensor force is required."

From the EFT perspective, a failure to reproduce nuclear matter observables should not be interpreted as showing that the low-energy potential is wrong, 
but that it is incomplete (e.g., many-body forces). This misconception still persists and has recently led to the conclusion that low-momentum two-nucleon interactions are "wrong" since they do not give saturation in nuclear matter and finite nuclei are overbound for lower cutoffs $[17,18]$. The missing physics that invalidates this conclusion is many-body forces, which were completely neglected in those studies.

In a low-energy effective theory, many-body forces are inevitable; the relevant question is how large they are. It is established beyond doubt that for all realistic potentials, a significant three-body force is required to describe light nuclei $[6,7,19,20]$. For variable-cutoff potentials, the three-body (and higher many-body) components of the potential also evolve with the resolution scale. A full RG evolution of the combined two- and three-body potential is not yet available, but can be approximated by fitting the three-body potential at each cutoff to the form of the leading-order $3 \mathrm{~N}$ force from chiral EFT. The first study of this sort revealed that the leading three-body components of the $3 \mathrm{~N}$ force become perturbative at lower cutoffs [21], which implies they are tractable in many-body calculations. In this paper, we apply these potentials to nuclear matter and study the convergence properties of low-momentum interactions.

The plan of this paper is as follows. In Section 2, a variable-cutoff potential is applied to the Born series for the two-nucleon amplitude in free space and as a function of density. The convergence properties as a function of cutoff are analyzed quantitatively using an approach introduced long ago by Weinberg. In Section 3, calculations of nuclear matter using the low-momentum two-nucleon interaction $V_{\text {low } k}$ with a corresponding leading-order $3 \mathrm{~N}$ force from chiral EFT are presented in the Hartree-Fock approximation and an approximation to second order. Nuclear saturation naturally arises at the Hartree-Fock level and becomes less cutoff dependent with the inclusion of the dominant second-order contributions. Our conclusions are summarized in Section 4 along with a plan for further calculations of nuclear matter.

\section{Perturbative ladders with low-momentum interactions}

In this section, we demonstrate that two sources of nonperturbative behavior in the particle-particle channel, namely "hard core" ${ }^{1}$ scattering and iterated tensor force contributions, can be rendered perturbative by using the RG to lower the momentum cutoff. We also show that, at sufficiently high density, Pauli blocking suppresses the nonperturbative physics due to bound or nearly-

1 We use "hard core" as a shorthand for the strong (but not infinite) short-range repulsion in conventional nuclear forces. 
bound states $[22,23]$. In the language of EFT, this means that the infraredenhancements that occur in the presence of such states in free space [24] are absent in nuclear matter.

In order to keep the presentation self-contained, we briefly review the RG method used to construct low-momentum NN interactions (" $V_{\text {low }} k$ ") starting from an arbitrary potential model $V_{\mathrm{NN}}[12,13,14]$. In a given partial wave, two-nucleon scattering is described by the $T$ matrix, which with standingwave boundary conditions and in our conventions $\left(\hbar=m_{\mathrm{N}}=1\right)$ is given by ${ }^{2}$

$$
T\left(k^{\prime}, k ; k^{2}\right)=V_{\mathrm{NN}}\left(k^{\prime}, k\right)+\frac{2}{\pi} \mathcal{P} \int_{0}^{\infty} q^{2} d q \frac{V_{\mathrm{NN}}\left(k^{\prime}, q\right) T\left(q, k ; k^{2}\right)}{k^{2}-q^{2}} .
$$

Here $\mathcal{P}$ denotes a principal value integration and the scattering phase shifts are given in terms of the diagonal $T$ matrix elements, $\tan \delta(k)=-k T\left(k, k ; k^{2}\right)$. Next, we define a low-momentum version of Eq. (1) in which a cutoff $\Lambda$ is imposed on loop integrals and the external legs of the interaction,

$$
T_{\text {low } k}\left(k^{\prime}, k ; k^{2}\right)=V_{\text {low } k}\left(k^{\prime}, k\right)+\frac{2}{\pi} \mathcal{P} \int_{0}^{\Lambda} q^{2} d q \frac{V_{\text {low } k}\left(k^{\prime}, q\right) T_{\text {low } k}\left(q, k ; k^{2}\right)}{k^{2}-q^{2}} .
$$

As the matching condition, we demand that the half-on-shell $T$ matrices are equivalent $T_{\text {low } k}\left(k^{\prime}, k ; k^{2}\right)=T\left(k^{\prime}, k ; k^{2}\right)$ for $k^{\prime}, k<\Lambda$ and all cutoffs. This ensures that $V_{\text {low } k}$ gives the same low-momentum two-nucleon observables as $V_{\mathrm{NN}}$ and has the advantage that it avoids energy-dependent interactions, which are inconvenient in many-body applications. The cutoff independence of the low-momentum $T$ matrix leads to an RG equation (for details see [14])

$$
\frac{d}{d \Lambda} V_{\text {low } k}\left(k^{\prime}, k\right)=\frac{2}{\pi} \frac{V_{\text {low } k}\left(k^{\prime}, \Lambda\right) T_{\text {low } k}\left(\Lambda, k ; \Lambda^{2}\right)}{1-(k / \Lambda)^{2}} .
$$

Whereas the $T$ matrices in Eqs. (1) and (2) are right-side half-on-shell, the $T$ matrix in the RG equation is left-side half-on-shell and is not RG invariant. This RG equation is asymmetric in $k$ and $k^{\prime}$, so it generates a non-hermitian $V_{\text {low } k}$. We avoid this by working with a symmetrized version of Eq. (3),

$$
\frac{d}{d \Lambda} V_{\text {low } k}\left(k^{\prime}, k\right)=\frac{1}{\pi}\left(\frac{V_{\text {low } k}\left(k^{\prime}, \Lambda\right) T_{\text {low } k}\left(\Lambda, k ; \Lambda^{2}\right)}{1-(k / \Lambda)^{2}}+\frac{T_{\text {low } k}\left(k^{\prime}, \Lambda ; \Lambda^{2}\right) V_{\text {low } k}(\Lambda, k)}{1-\left(k^{\prime} / \Lambda\right)^{2}}\right),
$$

which preserves the on-shell $T$ matrix (i.e., observables) and is equivalent to the Okubo hermitization of effective interaction theory. We note that $V_{\text {low }} k$ can be obtained by numerically integrating the RG equation with $V_{\mathrm{NN}}$ as the large-cutoff initial condition, or equivalently by Feshbach projection-operator techniques such as the Lee-Suzuki method (for details see $[13,14]$ ).

$\overline{2}$ This quantity, which is real, is sometimes referred to as the $K$ matrix. 


\subsection{Born series in free space vs. in medium}

We can now use the RG to study the convergence properties of free-space and in-medium $T$ matrices as we change the resolution scale $\Lambda$. In perturbation theory, the $T$ matrices are given by the Born series,

$$
T(E)=V+V G_{0}(E) V+V G_{0}(E) V G_{0}(E) V+\ldots,
$$

where the unperturbed propagator $G_{0}(E)$ is

$$
G_{0}(E)=\frac{1}{E-H_{0}} \quad(\text { free-space }), \quad G_{0}(E)=\frac{Q_{k_{\mathrm{F}}}}{E-H_{0}} \quad(\text { in-medium }),
$$

and $Q_{k_{\mathrm{F}}}$ is the Pauli-blocking operator that allows scattering only to unoccupied states above the Fermi momentum $k_{\mathrm{F}}$. In momentum space, the first and second Born terms are given by

$$
\begin{aligned}
& T^{(1)}\left(k^{\prime}, k ; E\right)=V\left(k^{\prime}, k\right), \\
& T^{(2)}\left(k^{\prime}, k ; E\right)=\frac{2}{\pi} \mathcal{P} \int q^{2} d q \frac{V\left(k^{\prime}, q\right) V(q, k)}{E-q^{2}} \quad \text { (free-space), } \\
& T^{(2)}\left(k^{\prime}, k ; E\right)=\frac{2}{\pi} \mathcal{P} \int q^{2} d q \frac{V\left(k^{\prime}, q\right) Q\left(q, P ; k_{\mathrm{F}}\right) V(q, k)}{E-q^{2}-P^{2} / 4} \quad \text { (in-medium), }
\end{aligned}
$$

where $P$ is the total pair momentum, and for S-waves the Pauli-blocking operator is given after angular integration by

$$
Q\left(q, P ; k_{\mathrm{F}}\right)=\left\langle q, P\left|Q_{k_{\mathrm{F}}}\right| q, P\right\rangle= \begin{cases}0 & \text { for } q<\sqrt{k_{\mathrm{F}}^{2}-P^{2} / 4} \\ 1 & \text { for } q>k_{\mathrm{F}}+P / 2 \\ \frac{q^{2}+P^{2} / 4-k_{\mathrm{F}}^{2}}{q P} & \text { otherwise. }\end{cases}
$$

In the above equations, $V\left(k^{\prime}, k\right)$ stands for either $V_{\text {low } k}$ or the input potential $V_{\mathrm{NN}}$, and the integration is from 0 to $\Lambda$ or 0 to $\infty$, respectively. For simplicity, we neglect self-energy effects and restrict our attention to total pair momentum $P=0$ in this section. The dependence on $P$ is generally very weak, and so our conclusions hold for non-zero $P$ as well.

The left panel of Fig. 1 shows the first- and second-order contributions to the diagonal $T$ matrix elements calculated from the Argonne $v_{18}$ potential in the ${ }^{1} \mathrm{~S}_{0}$ channel. It is evident that perturbative expansions of the free-space and in-medium $T$ matrices are not possible, as the second-order contribution is several times larger than the first-order contribution over all momenta considered. This divergence continues in higher orders. The strong repulsive core in the Argonne $v_{18}$ interaction, or any other conventional NN potential model, leads 

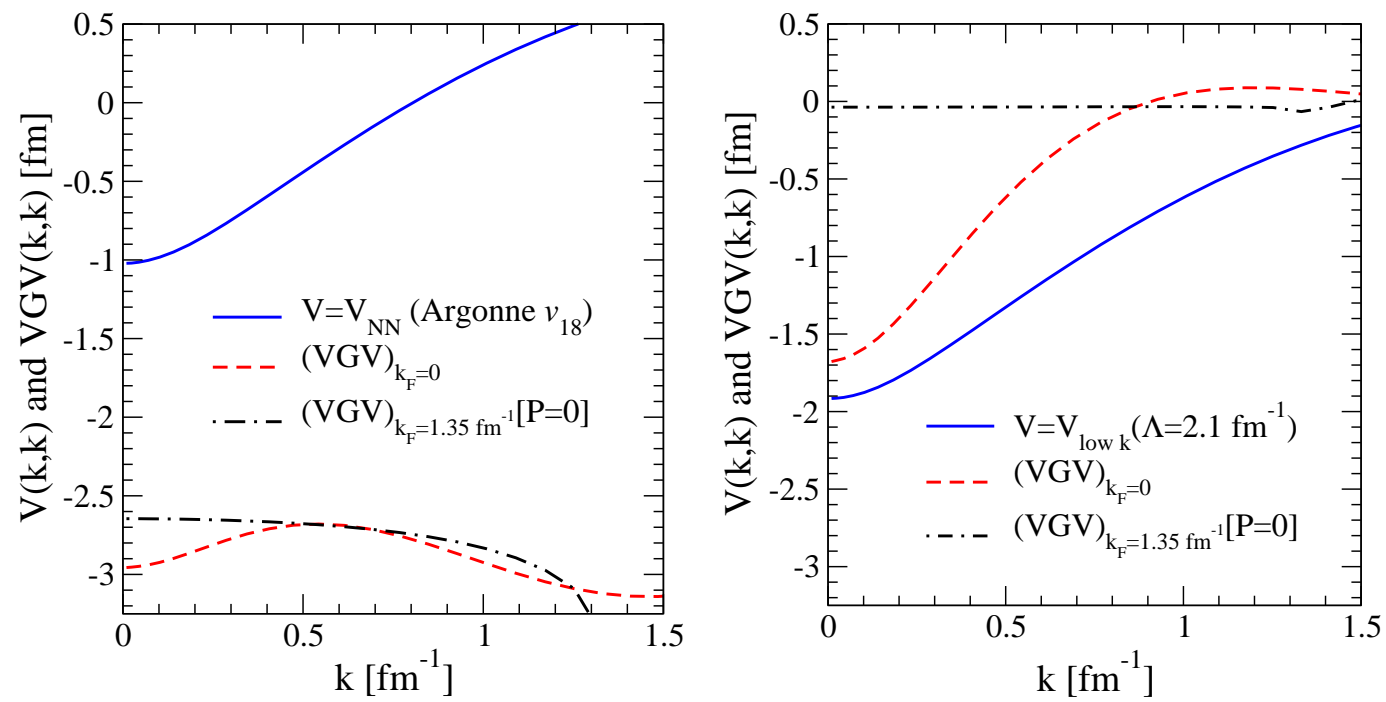

Fig. 1. Perturbative contributions to the diagonal ${ }^{1} \mathrm{~S}_{0} T$ matrices calculated with the Argonne $v_{18}$ potential (left panel) and with $V_{\text {low } k}$ for $\Lambda=2.1 \mathrm{fm}^{-1}$ (right panel). The first-order interactions are given by the solid lines. The second-order free-space (in-medium) contributions are denoted by the dashed (dot-dashed) lines. The in-medium contributions are evaluated at saturation density and for total pair momentum $P=0$. Here and in the following $V_{\text {low } k}$ is calculated from the Argonne $v_{18}$ potential.

to strong high-momentum matrix elements, and the momentum-independent nonperturbative behavior observed in Fig. 1 is a signature of "hard core" scattering. We emphasize that cores are not constrained by fits to low-energy two-nucleon data, and therefore "hard core" divergences of perturbation theory are not dictated by physics.

Pauli blocking does not change this picture, and in nuclear matter the secondorder contribution is comparable to the free-space one, as can be seen from Fig. 1. Therefore, "hard core" scattering always dominates. One might have expected that the second-order contribution is small at threshold in nuclear matter, since Pauli blocking suppresses the infrared enhancements that occur in free space at low energies. Clearly, this is not the case with conventional potential models due to the cores.

Referring to the right panel of Fig. 1, we find that the results obtained from $V_{\text {low } k}$ with $\Lambda=2.1 \mathrm{fm}^{-1}$ exhibit a very different pattern. We first observe that the second-order contributions to the amplitudes are smaller than the first-order Born term over the range of momenta considered. The secondorder free-space contribution is largest at zero energy due to the large ${ }^{1} \mathrm{~S}_{0}$ scattering length, ${ }^{3}$ while it decreases rapidly away from threshold, indicating that "hard core" scattering is absent. We emphasize that the diagonal free-

$\overline{3}$ With the normalization used here, the contributions at $k=0$ will sum to the ${ }^{1} \mathrm{~S}_{0}$ neutron-proton scattering length of $a_{\mathrm{S}_{0}}=-23.7 \mathrm{fm}$. 


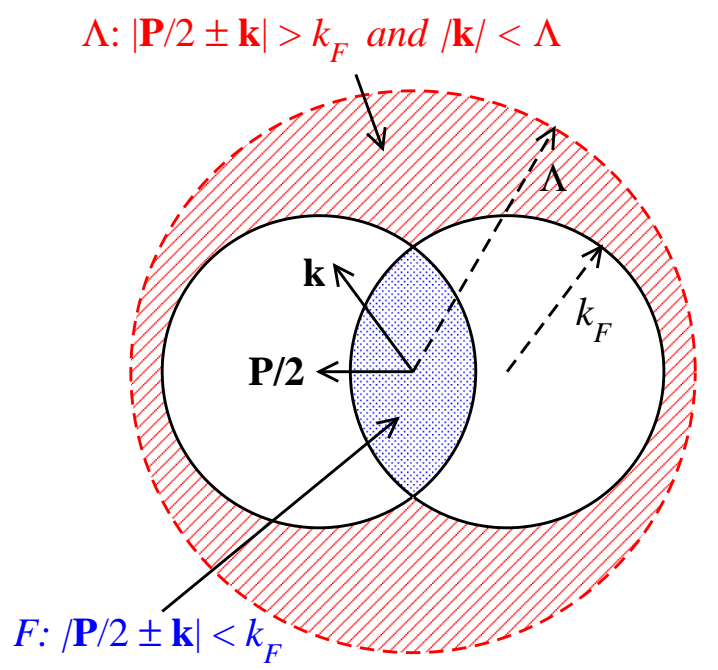

Fig. 2. Pauli-allowed intermediate-state phase space (hatched region " $\Lambda$ ") for scattering in the particle-particle channel. Nucleons are initially inside the Fermi sea (shaded region "F") and interactions are restricted to momenta below the cutoff $\Lambda$.

space $T$ matrix elements obtained from an exact solution using either the Argonne $v_{18}$ potential or $V_{\text {low } k}$ are identical up to $330 \mathrm{MeV}$ lab energy. This demonstrates that the convergence behavior of the Born series in free space is strongly scale-dependent, with lower cutoffs being advantageous.

For the in-medium calculation, we find the striking result that the second-order contribution is very small over all momenta. This dramatic improvement of in-medium convergence can be easily understood from a simple phase-space argument. In the present calculation for total pair momentum $P=0$, the limits of the intermediate-state integrals in Eq. (7) are from $k_{\mathrm{F}}$ to $\Lambda$ for $V_{\text {low } k}$. In contrast, the loop integrals for conventional potentials require large upper limits of $15-20 \mathrm{fm}^{-1}$. Therefore, the restriction of phase-space substantially reduces the size of higher-order corrections. This reduction is also aided by weaker interactions at higher momenta in the dominant S-waves.

This phase-space argument also holds for general $P \neq 0$. In Fig. 2, we show the allowed phase space for the general case, where a pair of particles with total momentum $P$ initially in the Fermi sea (the shaded region " $F$ ") scatters to Pauli-allowed intermediate state (the hatched region " $\Lambda$ "). It is clear from Fig. 2 that lowering the cutoff suppresses the intermediate-state phase space for $P \neq 0$ case as well. When $P$ is very close to $2 k_{\mathrm{F}}$ the suppression is less extreme, but a corresponding $V_{\text {low } k} G V_{\text {low } k}$ curve as in Fig. 1 is still less than $0.3 \mathrm{fm}$ in magnitude throughout the range of $k$.

However, the cutoff also has to be greater than the largest relative momentum with non-negligible contributions to the observable of interest. It is evident from Fig. 2 that for large total pair momentum (e.g., $P=2 k_{\mathrm{F}}$ ), cutoffs $\Lambda<2 k_{\mathrm{F}}$ cut into the Fermi spheres. For general interactions, taking the cutoff too small 
could complicate the task of maintaining cutoff-independent observables. This is because the reshuffling of contributions from loop integrals to the effective interaction in the $\mathrm{RG}$ equation is defined in free space. In this paper we take a pragmatic approach to this issue. Energy-per-particle results for $\Lambda<2 k_{\mathrm{F}}$ are found to be very weakly dependent on the cutoff, which indicates that such configurations contribute negligibly to bulk properties. ${ }^{4}$ Moreover, region "F" vanishes as $P \rightarrow 2 k_{\mathrm{F}}$ and the average pair momentum in the Fermi sea is $P_{\mathrm{av}}=\sqrt{6 / 5} k_{\mathrm{F}}$. Therefore, it is likely that the limit $\Lambda \geqslant 2 k_{\mathrm{F}}$ set by the $P=2 k_{\mathrm{F}}$ configurations is too conservative.

In summary, we find that the perturbative convergence of two-nucleon scattering is improved dramatically when we use the RG to evolve the interaction down to lower cutoffs. The resulting $V_{\text {low } k}$ is by construction phase-shift equivalent to the input potential, but $V_{\text {low } k}$ removes the difficulties due to repulsive cores. In-medium ladders become perturbative because lower cutoffs restrict the intermediate-state phase-space integrations, and because NN scattering in the dominant S-waves is weaker at higher momenta.

\subsection{Weinberg eigenvalue analysis}

The preceding conclusions can be made rigorous by adopting a method introduced by Weinberg in Ref. [26] that provides quantitative conditions for the perturbative convergence of the $T$ matrix. This allows us to identify the cutoffs at which "hard core" scattering and iterated tensor contributions become perturbative. Moreover, the analysis shows that Pauli-blocking removes nonperturbative behavior due to low-energy bound or nearly-bound states. Thus, we will establish as a main result of this paper that the in-medium $T$ matrix is perturbative over a wide range of cutoffs and densities.

Following Weinberg [26], we study the spectrum of the operator $G_{0}(z) V$ for complex energies $z$

$$
G_{0}(z) V\left|\Psi_{\nu}(z)\right\rangle=\eta_{\nu}(z)\left|\Psi_{\nu}(z)\right\rangle
$$

where the index $\nu$ labels the discrete eigenvalues and eigenvectors. As demonstrated in [26], the perturbative Born series for $T(z)$ diverges if and only if there is an eigenvalue with $\left|\eta_{\nu}(z)\right| \geqslant 1$. The necessity of this condition is clear, since

$$
\sum_{n=0}^{\infty} V\left(G_{0}(z) V\right)^{n}\left|\Psi_{\nu}(z)\right\rangle=\sum_{n=0}^{\infty}\left(\eta_{\nu}(z)\right)^{n} V\left|\Psi_{\nu}(z)\right\rangle
$$

We also note that observables for which relative momenta $k>2 \mathrm{fm}^{-1}$ contribute significantly will be model dependent. This is the case, for example, for P-wave pairing gaps for neutron Fermi momenta $k_{\mathrm{F}}>2 \mathrm{fm}^{-1}$ [25]. 
and thus

$$
T(z)\left|\Psi_{\nu}(z)\right\rangle=\left(1+\eta_{\nu}(z)+\eta_{\nu}(z)^{2}+\ldots\right) V\left|\Psi_{\nu}(z)\right\rangle
$$

diverges for $\left|\eta_{\nu}(z)\right| \geqslant 1$. Furthermore, Weinberg also demonstrates that the rate of perturbative convergence is controlled by the largest $\left|\eta_{\nu}(z)\right|$, with smaller values implying faster convergence.

A rearrangement of Eq. (9) gives a simple interpretation of the eigenvalues $\eta_{\nu}(z)$ in terms of the Schrödinger equation,

$$
\left(H_{0}+\frac{1}{\eta_{\nu}(z)} V\right)\left|\Psi_{\nu}(z)\right\rangle=z\left|\Psi_{\nu}(z)\right\rangle .
$$

The eigenvalue $\eta_{\nu}(z)$ can thus be viewed as an energy-dependent coupling constant that must divide $V$ to produce a solution to the Schrödinger equation at energy $z$. If $V$ supports a bound state at $z=B<0$, then there is some $\nu$ with $\eta_{\nu}(B)=1$, which implies a divergence of the Born series for nearby energies. However, what matters for convergence at a given energy $z$ is not simply the presence of nearby physical bound states, but rather the complete set of eigenstates that can be shifted to $z$ when the interaction is divided by $\eta_{\nu}(z)$. At negative energies, a purely attractive $V$ gives positive $\eta_{\nu}(z)$ values, while a purely repulsive $V$ gives negative values, as the sign of the interaction must be flipped to support a bound state. For this reason, we follow convention and refer to negative eigenvalues as repulsive and positive ones as attractive. In the case of conventional NN interactions, the repulsive core generates at least one large and negative eigenvalue that causes the Born series to diverge.

We apply the spectral analysis to the coupled ${ }^{3} \mathrm{~S}_{1}-{ }^{3} \mathrm{D}_{1}$ channel and show in Fig. 3 the evolution of the two largest Weinberg eigenvalues evaluated at the deuteron pole $z=B_{d}$, as we lower the cutoff. From the free-space curves (solid lines), we observe that the repulsive eigenvalue associated with the core and the tensor force is initially large but decreases with cutoff, with $\left|\eta_{\nu}\left(B_{d}\right)\right|<1$ for $\Lambda<3.5 \mathrm{fm}^{-1}$. In contrast, the physical divergence corresponding to the deuteron pole remains invariant, $\eta_{\nu}\left(B_{d}\right)=1$, as it should. This suggests that the nonperturbative behavior due to the strong core and strong tensor force is only present for large-cutoff interactions.

The finite-density curves (dashed lines) show a similar dependence on cutoff, but now the nonperturbative attractive eigenvalue associated with the deuteron in free space is tamed by Pauli-blocking effects. This result is general, as illustrated by Fig. 4, where we show the density dependence of the Weinberg eigenvalues for various cutoffs. As the density increases, Pauli-blocking drives the largest attractive Weinberg eigenvalue to a perturbative regime.

It is important to note that the repulsive eigenvalue is significantly reduced 


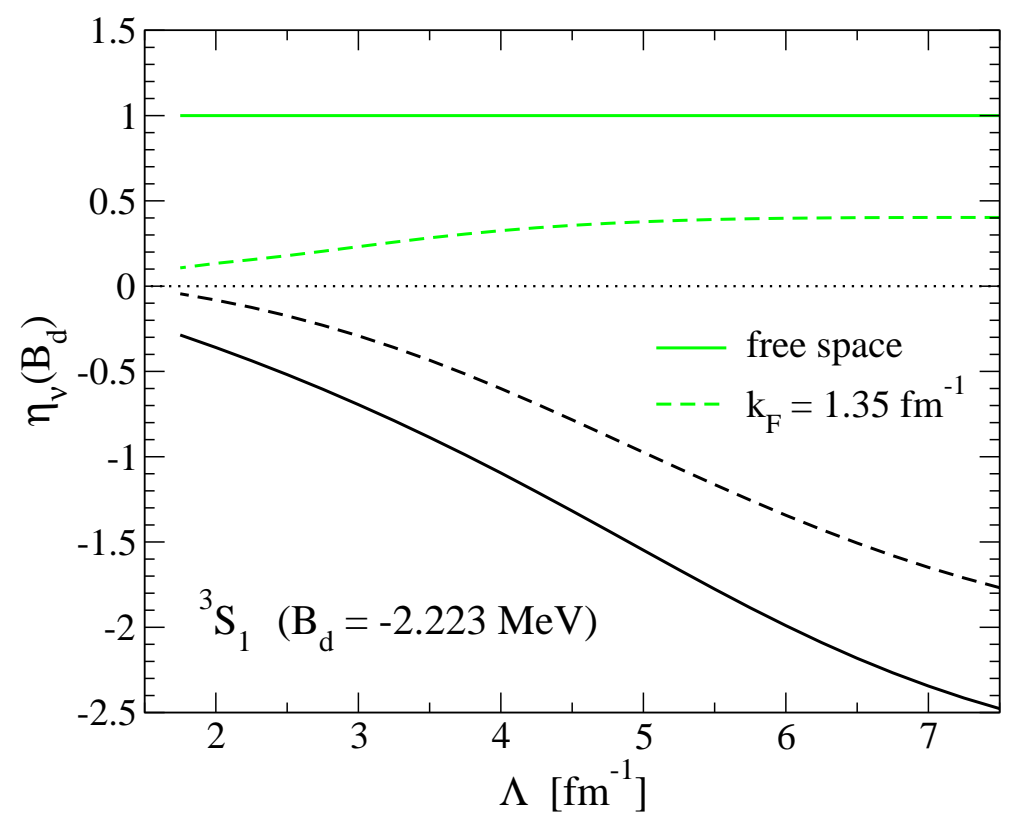

Fig. 3. Evolution of the two largest Weinberg eigenvalues with $\Lambda$ in the coupled ${ }^{3} \mathrm{~S}_{1}-{ }^{3} \mathrm{D}_{1}$ channel in free-space (solid) and at saturation density (dashed). The lighter curves correspond to attractive eigenvalues and the darker curves to repulsive eigenvalues.

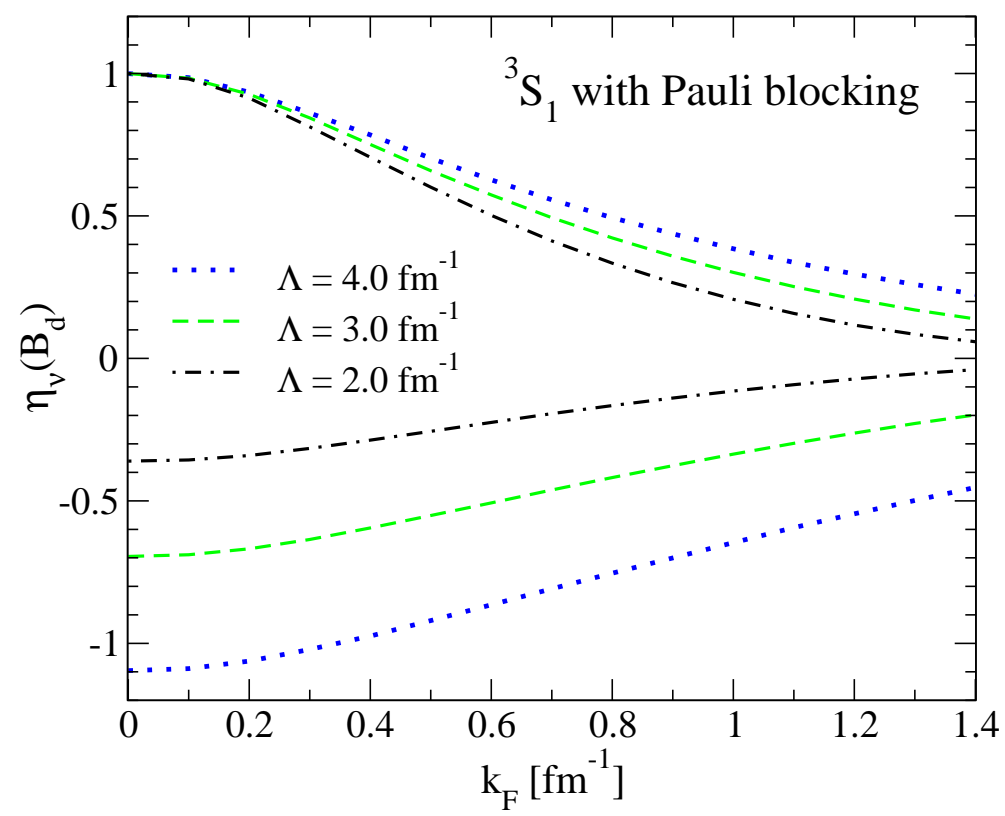

Fig. 4. Dependence on density of the two largest Weinberg eigenvalues in the coupled ${ }^{3} \mathrm{~S}_{1}-{ }^{3} \mathrm{D}_{1}$ channel.

when the cutoff is lowered from $\Lambda=3 \mathrm{fm}^{-1}$ to $\Lambda=2 \mathrm{fm}^{-1}$. Second-order tensor contributions strongly excite intermediate-state momenta peaked about $k \approx 2.5-3.0 \mathrm{fm}^{-1}$ in nuclear matter [27]. Consequently, when the cutoff is lowered below this range, the strength of the tensor force decreases, leading to a smaller Weinberg eigenvalue. Chiral EFT interactions have Weinberg eigen- 
values similar to the $\Lambda=3 \mathrm{fm}^{-1}$ curves, and thus their in-medium convergence properties can be improved by using the RG to run their cutoff to lower values.

We therefore reach a promising conclusion: Large-cutoff sources of nonperturbative behavior can be eliminated using the RG to lower the momentum cutoff, while the physical source of nonperturbative behavior due to bound and nearly-bound states is suppressed in the medium by the Pauli principle. For simplicity, the above analysis was for NN interactions only, but as we show in the next section, a consistent treatment of many-nucleon forces does not change the general conclusion.

\section{$3 \quad$ Nuclear matter results}

It is evident from the preceding section that for nuclear interactions with large cutoffs, the strong cores and tensor force necessitate a resummation of particle-particle scattering in nuclear matter. This resummation is typically carried out in the Brueckner approach, which sums iterated particle-particle scattering within a self-consistent mean-field (for reviews see $[28,29]$ ).

In Fig. 5, we show the particle-particle contributions to the energy of symmetric matter in perturbation theory. The calculations are carried out using a continuous single-particle spectrum with the effective mass approximation, and all intermediate-state phase-space integrations are angle-averaged. Fig. 5 demonstrates that for large-cutoff interactions, such as the Argonne $v_{18}$ potential, iterated particle-particle scattering is nonperturbative. We repeat that this behavior is not required by nucleon-nucleon scattering data, and may be viewed as an artifact of the large cutoff in the Argonne $v_{18}$ potential. In stark contrast, for the low-momentum interaction $V_{\text {low } k}$ with $\Lambda=2.1 \mathrm{fm}^{-1}$ we observe that particle-particle ladders are perturbative in nuclear matter. These results are due to restricted phase space and follow from the behavior of the Weinberg eigenvalues, as discussed in the previous section.

With two-nucleon low-momentum interactions only, particle-particle correlations do not lead to saturation within the density range where nuclear forces are well-constrained experimentally. We also note that for two-nucleon forces with cores, saturation occurs at Fermi momenta $k_{\mathrm{F}}>1.5 \mathrm{fm}^{-1}$ in the Brueckner approach $[28,29,30]$, in contrast to the empirical value of $k_{\mathrm{F}}=1.35 \mathrm{fm}^{-1}$. Such calculations overbind nuclear matter by several $\mathrm{MeV}$ relative to the empirical value of $E / A=-16 \mathrm{MeV}$. Moreover, it is well-established that $3 \mathrm{~N}$ interactions present important contributions in light nuclei $[7,19,20]$. In Ref. [21], we showed that $3 \mathrm{~N}$ contributions are smaller for low-momentum interactions, and that $3 \mathrm{~N}$ forces are required to guarantee regulator-independent results. Therefore, it is imperative that $3 \mathrm{~N}$ forces are included in calculations of nuclear 


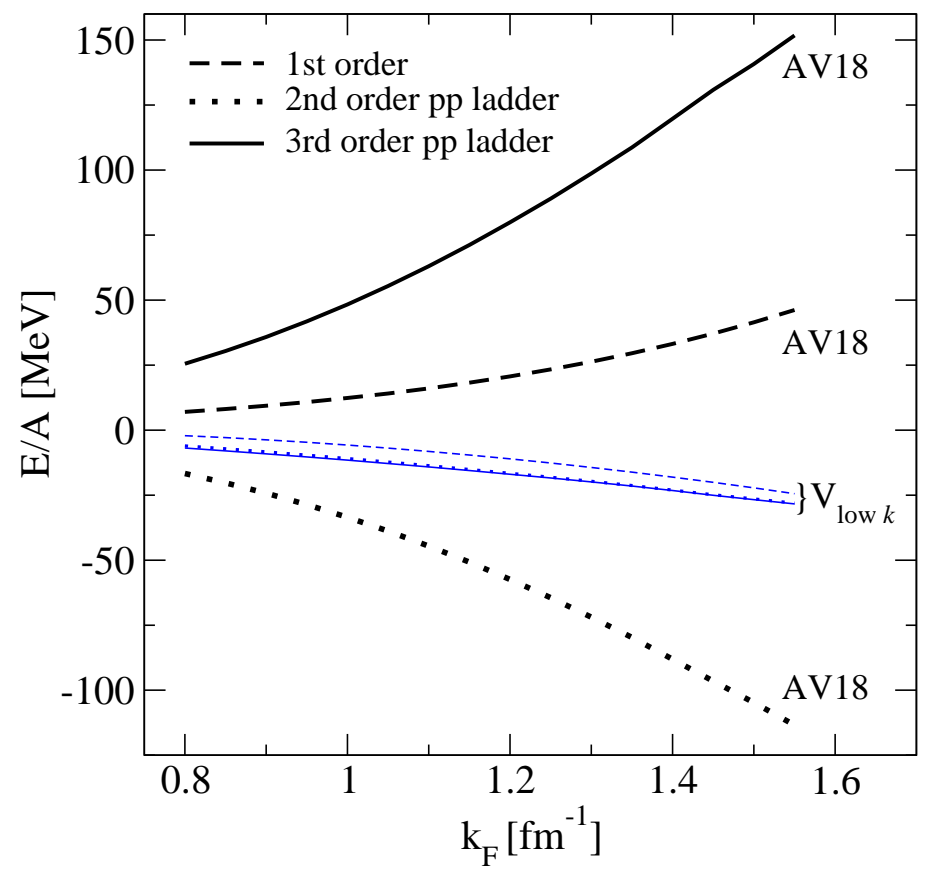

Fig. 5. Particle-particle contributions to the energy per particle in symmetric nuclear matter for the Argonne $v_{18}$ potential (thick lines) and the low-momentum interaction $V_{\text {low } k}$ with $\Lambda=2.1 \mathrm{fm}^{-1}$ (thin lines).

matter.

As discussed in the introduction, an RG evolution of the combined two- and three-nucleon interactions is not yet available. However, we have argued that this evolution can be approximated by fitting the leading-order $3 \mathrm{~N}$ force from chiral EFT at each cutoff to be used with $V_{\text {low } k}$ [21]. This is motivated by the fact that $V_{\text {low } k}$ becomes independent of the starting two-nucleon potential as the cutoff is lowered to $\Lambda \lesssim 2 \mathrm{fm}^{-1}$. The same model-independent $V_{\text {low } k}$ is obtained by evolving a chiral $\mathrm{N}^{3} \mathrm{LO}$ two-nucleon interaction to smaller cutoffs. The RG evolution induces contact interactions which are of higher order in a chiral expansion and are necessary to maintain cutoff-independent two-nucleon observables. This indicates that $V_{\text {low } k}$ effectively parameterizes a chiral EFT interaction with higher-order contact terms, and supports our assumption that the low-momentum $3 \mathrm{~N}$ interaction corresponding to $V_{\text {low }} k$ is well-approximated by the leading-order chiral $3 \mathrm{~N}$ force.

Therefore, we have augmented $V_{\text {low } k}$ with the leading-order chiral $3 \mathrm{~N}$ force [21]. The latter contains a long-range $2 \pi$-exchange part $V_{c}$, an intermediate-range $1 \pi$-exchange part $V_{D}$ and a short-range contact interaction $V_{E}$ [31,32]. The $2 \pi$-exchange interaction is given by

$$
V_{c}=\frac{1}{2}\left(\frac{g_{A}}{2 f_{\pi}}\right)^{2} \sum_{i \neq j \neq k} \frac{\left(\boldsymbol{\sigma}_{i} \cdot \mathbf{q}_{i}\right)\left(\boldsymbol{\sigma}_{j} \cdot \mathbf{q}_{j}\right)}{\left(q_{i}^{2}+m_{\pi}^{2}\right)\left(q_{j}^{2}+m_{\pi}^{2}\right)} F_{i j k}^{\alpha \beta} \tau_{i}^{\alpha} \tau_{j}^{\beta}
$$


where $\mathbf{q}_{i}=\mathbf{k}_{i}^{\prime}-\mathbf{k}_{i}$ denotes the difference of initial and final nucleon momenta and

$$
F_{i j k}^{\alpha \beta}=\delta^{\alpha \beta}\left[-\frac{4 c_{1} m_{\pi}^{2}}{f_{\pi}^{2}}+\frac{2 c_{3}}{f_{\pi}^{2}} \mathbf{q}_{i} \cdot \mathbf{q}_{j}\right]+\sum_{\gamma} \frac{c_{4}}{f_{\pi}^{2}} \epsilon^{\alpha \beta \gamma} \tau_{k}^{\gamma} \boldsymbol{\sigma}_{k} \cdot\left(\mathbf{q}_{i} \times \mathbf{q}_{j}\right),
$$

while the $1 \pi$-exchange and contact interactions are given respectively by

$$
\begin{aligned}
V_{D} & =-\frac{g_{A}}{8 f_{\pi}^{2}} \frac{c_{D}}{f_{\pi}^{2} \Lambda_{\chi}} \sum_{i \neq j \neq k} \frac{\boldsymbol{\sigma}_{j} \cdot \mathbf{q}_{j}}{q_{j}^{2}+m_{\pi}^{2}}\left(\boldsymbol{\tau}_{i} \cdot \boldsymbol{\tau}_{j}\right)\left(\boldsymbol{\sigma}_{i} \cdot \mathbf{q}_{j}\right), \\
V_{E} & =\frac{c_{E}}{2 f_{\pi}^{4} \Lambda_{\chi}} \sum_{i \neq j \neq k}\left(\boldsymbol{\tau}_{j} \cdot \boldsymbol{\tau}_{k}\right) .
\end{aligned}
$$

In applying Eqs. (13)-(16), we use $g_{A}=1.29, f_{\pi}=92.4 \mathrm{MeV}$ and $m_{\pi}=$ 138.04 MeV and the $c_{i}$ constants extracted by the Nijmegen group in a partial wave analysis with chiral $2 \pi$-exchange [33]. These are $c_{1}=-0.76 \mathrm{GeV}^{-1}$, $c_{3}=-4.78 \mathrm{GeV}^{-1}$ and $c_{4}=3.96 \mathrm{GeV}^{-1}$. The fit values for the $c_{D}$ and $c_{E}$ low-energy constants are tabulated in Ref. [21] for $\Lambda_{\chi}=700 \mathrm{MeV}$.

Motivated by the above discussion, we proceed to calculate the energy per particle in symmetric nuclear matter from $V_{\text {low } k}$ and $V_{3 \mathrm{~N}}$ in many-body perturbation theory. The $V_{\text {low } k}$ and $V_{3 \mathrm{~N}}$ Hartree-Fock contributions are given by

$$
\begin{aligned}
\frac{E_{V_{\text {low } k}}^{(1)}}{V} & =\frac{1}{2} \operatorname{Tr}_{\sigma_{1}, \tau_{1}} \operatorname{Tr}_{\sigma_{2}, \tau_{2}} \int \frac{d \mathbf{k}_{1}}{(2 \pi)^{3}} \int \frac{d \mathbf{k}_{2}}{(2 \pi)^{3}} n_{k_{1}} n_{k_{2}}\left\langle 12\left|V_{\text {low } k}\left(1-P_{12}\right)\right| 12\right\rangle \\
\frac{E_{V_{3 \mathrm{~N}}}^{(1)}}{V} & =\frac{1}{6} \operatorname{Tr}_{\sigma_{1}, \tau_{1}} \operatorname{Tr}_{\sigma_{2}, \tau_{2}} \operatorname{Tr}_{\sigma_{3}, \tau_{3}} \int \frac{d \mathbf{k}_{1}}{(2 \pi)^{3}} \int \frac{d \mathbf{k}_{2}}{(2 \pi)^{3}} \int \frac{d \mathbf{k}_{3}}{(2 \pi)^{3}} n_{k_{1}} n_{k_{2}} n_{k_{3}} \\
& \times f_{\mathrm{R}}^{2}(p, q)\left\langle 123\left|V_{3 \mathrm{~N}} \mathcal{A}_{123}\right| 123\right\rangle
\end{aligned}
$$

where the $n_{k_{i}}$ denote zero temperature Fermi-Dirac distributions, $P_{12}$ is the exchange operator for spin, isospin and momenta of nucleons 1 and 2 , and $V$ is the volume. Moreover, the momentum-conserving delta functions are not included in the $\mathrm{NN}$ and $3 \mathrm{~N}$ matrix elements. For the $3 \mathrm{~N}$ contribution,

$$
f_{\mathrm{R}}(p, q)=\exp \left[-\left(\frac{p^{2}+3 q^{2} / 4}{\Lambda^{2}}\right)^{4}\right]
$$

is the regulator used in the $3 \mathrm{~N}$ force fits in Ref. [21] and $p$ and $q$ are Jacobi momenta. Exchange terms are included by means of the antisymmetrizer

$$
\begin{aligned}
\mathcal{A}_{123} & =\left(1+P_{12} P_{23}+P_{13} P_{23}\right)\left(1-P_{23}\right) \\
& =1-P_{12}-P_{13}-P_{23}+P_{12} P_{23}+P_{13} P_{23}
\end{aligned}
$$

where the direct, single-exchange and double-exchange contributions are apparent in Eq. (21). The regulator $f_{\mathrm{R}}(p, q)$ is totally symmetric when expressed 
in the nucleon momenta $\mathbf{k}_{i}$, and thus all exchange terms contain the same regulator as the direct contribution.

For symmetric nuclear matter, we obtain the following Hartree-Fock contributions from the leading-order chiral $3 \mathrm{~N}$ force. For the contact part $\left(V_{E}\right)$ and the $1 \pi$-exchange $3 \mathrm{~N}$ interaction $\left(V_{D}\right)$ we have

$$
\begin{aligned}
\frac{E_{E}^{(1)}}{A} & =-\frac{9}{(2 \pi)^{4}} \frac{c_{E}}{f_{\pi}^{4} \Lambda_{\chi} k_{\mathrm{F}}^{3}} \int_{0}^{2 k_{\mathrm{F}}} P^{2} d P \int_{0}^{\sqrt{k_{\mathrm{F}}^{2}-\frac{P^{2}}{4}}} p^{2} d p \int_{0}^{k_{\mathrm{F}}+\frac{P}{2}} q^{\prime 2} d q^{\prime} \\
& \times F\left(p, P ; k_{\mathrm{F}}\right) G\left(q^{\prime}, P ; k_{\mathrm{F}}\right) f_{\mathrm{R}}^{2}\left(p, 2 q^{\prime} / 3\right), \\
\frac{E_{D}^{(1)}}{A} & =\frac{9}{(2 \pi)^{4}} \frac{g_{A} c_{D}}{4 f_{\pi}^{4} \Lambda_{\chi} k_{\mathrm{F}}^{3}} \int_{0}^{2 k_{\mathrm{F}}} P^{2} d P \int_{0}^{\sqrt{k_{\mathrm{F}}^{2}-\frac{P^{2}}{4}}} p^{2} d p \int_{0}^{k_{\mathrm{F}}+\frac{P}{2}} q^{\prime 2} d q^{\prime} \frac{4 p^{2}}{4 p^{2}+m_{\pi}^{2}} \\
& \times F\left(p, P ; k_{\mathrm{F}}\right) G\left(q^{\prime}, P ; k_{\mathrm{F}}\right) f_{\mathrm{R}}^{2}\left(p, 2 q^{\prime} / 3\right),
\end{aligned}
$$

where $F$ and $G$ are phase-space functions

$$
\begin{aligned}
F\left(p, P ; k_{\mathrm{F}}\right) & = \begin{cases}2 & \text { for } p<k_{\mathrm{F}}-P / 2 \\
2 \frac{k_{\mathrm{F}}^{2}-p^{2}-P^{2} / 4}{p P} & \text { otherwise }\end{cases} \\
G\left(q^{\prime}, P ; k_{\mathrm{F}}\right) & = \begin{cases}2 & \text { for } q^{\prime}<k_{\mathrm{F}}-P / 2 \\
\frac{k_{\mathrm{F}}^{2}-\left(q^{\prime}-P / 2\right)^{2}}{q^{\prime} P} & \text { otherwise. }\end{cases}
\end{aligned}
$$

It is useful to have simple estimates for these contributions. If the regulator is approximated by unity over the integration region, one has $E_{E}^{(1)} / A=$ $-c_{E} k_{\mathrm{F}}^{6} /\left(12 \pi^{4} f_{\pi}^{4} \Lambda_{\chi}\right)$. This is reasonable because of the high power in the exponential regulator $f_{\mathrm{R}}$. We further note that in the chiral limit, $m_{\pi} \rightarrow 0$, the ratio of $D$ - to $E$-term contributions is $E_{D}^{(1)} / E_{E}^{(1)}=-g_{A} c_{D} / 4 c_{E}$. This is an overestimate for finite pion mass by $\sim m_{\pi}^{2} / k_{\mathrm{F}}^{2} \approx 25 \%$. 
Table 1

\begin{tabular}{c|cc} 
& $c_{D}$ & $c_{E}$ \\
\hline 1.6 & 2.080 & 0.230 \\
1.9 & -1.225 & -0.405 \\
2.1 & -2.062 & -0.625 \\
2.3 & -2.785 & -0.822
\end{tabular}

Values for the low-energy couplings $c_{D}$ and $c_{E}$ adjusted to the ${ }^{3} \mathrm{H}$ and ${ }^{4} \mathrm{He}$ binding energies in [21]. As discussed in the text, the couplings for $\Lambda=2.1 \mathrm{fm}^{-1}$ and $\Lambda=$ $2.3 \mathrm{fm}^{-1}$ have been interpolated.

The remaining $2 \pi$-exchange $3 \mathrm{~N}$ interaction leads to

$$
\begin{aligned}
\frac{E_{c}^{(1)}}{A} & =\frac{9}{(2 \pi)^{5}} \frac{g_{A}^{2}}{k_{\mathrm{F}}^{3}} \int_{0}^{2 k_{\mathrm{F}}} P^{2} d P \int_{0}^{\sqrt{k_{\mathrm{F}}^{2}-\frac{P^{2}}{4}}} p^{2} d p \int_{0}^{k_{\mathrm{F}}+\frac{P}{2}} q^{\prime 2} d q^{\prime} \int_{-1}^{1} d \cos \theta_{\mathbf{p}} \int_{-1}^{1} d \cos \theta_{\mathbf{q}^{\prime}} \int_{0}^{2 \pi} d \varphi \\
& \times \mathcal{F}\left(p, P ; k_{\mathrm{F}}\right) \mathcal{G}\left(q^{\prime}, P ; k_{\mathrm{F}}\right) f_{\mathrm{R}}^{2}\left(p, 2 q^{\prime} / 3\right) \\
& \times\left[-\frac{c_{1} m_{\pi}^{2}}{f_{\pi}^{4}}\left(\frac{\mathbf{k}_{12} \cdot \mathbf{k}_{23}}{\left(k_{12}^{2}+m_{\pi}^{2}\right)\left(k_{23}^{2}+m_{\pi}^{2}\right)}+2 \frac{k_{12}^{2}}{\left(k_{12}^{2}+m_{\pi}^{2}\right)^{2}}\right)\right. \\
& +\frac{c_{3}}{2 f_{\pi}^{4}}\left(\frac{\left(\mathbf{k}_{12} \cdot \mathbf{k}_{23}\right)^{2}}{\left(k_{12}^{2}+m_{\pi}^{2}\right)\left(k_{23}^{2}+m_{\pi}^{2}\right)}-2 \frac{k_{12}^{4}}{\left(k_{12}^{2}+m_{\pi}^{2}\right)^{2}}\right) \\
& \left.-\frac{c_{4}}{2 f_{\pi}^{4}}\left(\frac{\left(\mathbf{k}_{12} \times \mathbf{k}_{23}\right)^{2}}{\left(k_{12}^{2}+m_{\pi}^{2}\right)\left(k_{23}^{2}+m_{\pi}^{2}\right)}\right)\right],
\end{aligned}
$$

where $\mathbf{k}_{i j}=\mathbf{k}_{i}-\mathbf{k}_{j}$ and the terms with $\mathbf{k}_{12}$ only are single-exchanges. Due to the angular dependence of the $2 \pi$-exchange $3 \mathrm{~N}$ interaction, the angular integrations do not simplify and the phase-space restrictions are

$$
\begin{aligned}
& \mathcal{F}\left(p, P ; k_{\mathrm{F}}\right)= \begin{cases}1 & \text { for } p<k_{\mathrm{F}}-P / 2 \\
\left|\cos \theta_{\mathbf{p}}\right| \leqslant \frac{k_{\mathrm{F}}^{2}-p^{2}-P^{2} / 4}{p P} & \text { otherwise }\end{cases} \\
& \mathcal{G}\left(q^{\prime}, P ; k_{\mathrm{F}}\right)= \begin{cases}1 & \text { for } q^{\prime}<k_{\mathrm{F}}-P / 2 \\
\cos \theta_{\mathbf{q}^{\prime}} \leqslant \frac{k_{\mathrm{F}}^{2}-q^{\prime 2}-P^{2} / 4}{q^{\prime} P} & \text { otherwise. }\end{cases}
\end{aligned}
$$

When the regulator is neglected, the $1 \pi$ - and $2 \pi$-exchange $3 \mathrm{~N}$ force contributions can be evaluated analytically or can be reduced to one-dimensional integrals. We give the corresponding expressions in the Appendix.

We emphasize that the parameters for the $3 \mathrm{~N}$ force were adjusted to the ${ }^{3} \mathrm{H}$ and ${ }^{4} \mathrm{He}$ binding energies in [21], and therefore the results presented in this section are predictions. To obtain results with $\Lambda=2.1 \mathrm{fm}^{-1}$ and $\Lambda=2.3 \mathrm{fm}^{-1}$, 

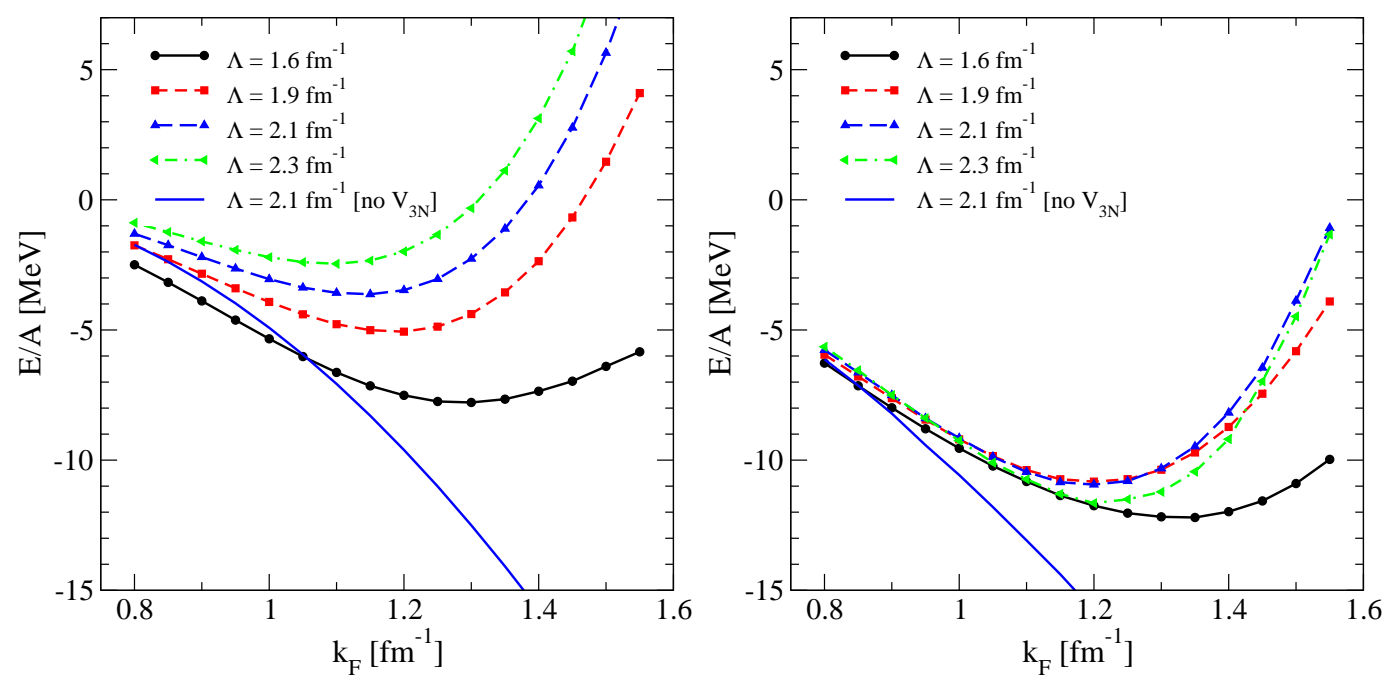

Fig. 6. Hartree-Fock (left figure) and Hartree-Fock plus dominant second-order contributions (right figure) calculated from $V_{\text {low } k}$ and $V_{3 \mathrm{~N}}$ for various cutoffs. Details of the approximate second-order calculations are given in the text.

we have interpolated between the smoothly-varying $c_{D}$ and $c_{E}$ fit values. ${ }^{5}$ The values for the low-energy couplings $c_{D}$ and $c_{E}$ are listed in Table 1. Finally, we note that all Hartree contributions vanish for the leading-order chiral $3 \mathrm{~N}$ interaction due to their spin-isospin structure.

In Fig. 6, we present Hartree-Fock results for the energy per particle in symmetric nuclear matter calculated from $V_{\text {low } k}$ and $V_{3 \mathrm{~N}}$ for various cutoffs. The remaining integrals in Eqs. (22), (23), and (26) were calculated numerically. The Hartree-Fock results show that nuclear saturation is due to $3 \mathrm{~N}$ forces when model-independent, low-momentum NN interactions are used. As can be seen from the $V_{\text {low } k}$ results in Fig. 6, the effects of $3 \mathrm{~N}$ forces is very small at low densities $k_{\mathrm{F}} \lesssim 0.8 \mathrm{fm}^{-1}$. At the Hartree-Fock level, we find a minimum of $E / A \approx-(2.5-8) \mathrm{MeV}$ for Fermi momenta $k_{\mathrm{F}} \approx(1.1-1.3) \mathrm{fm}^{-1}$ over the cutoff range considered. It is quite promising that nuclear matter saturates in the Hartree-Fock approximation for low-momentum NN and 3N interactions, as conventional NN and $3 \mathrm{~N}$ interactions require complicated nonperturbative treatments to achieve binding. However, we have not yet formulated a power counting appropriate for finite density that prescribes these ingredients at leading order.

As can be seen from Table 2 , the dominant $3 \mathrm{~N}$ contributions are due to the $2 \pi$-exchange interaction. For $k_{\mathrm{F}}=1.2 \mathrm{fm}^{-1}$ and $\Lambda=1.9 \mathrm{fm}^{-1}$, the $3 \mathrm{~N}$ HartreeFock expectation values are $E_{E}^{(1)} / A=1.2 \mathrm{MeV}, E_{D}^{(1)} / A=-0.8 \mathrm{MeV}$ and $E_{c}^{(1)} / A=5.6 \mathrm{MeV}$. The repulsive single-exchange $c$-terms are more than a factor two larger than the attractive double-exchanges. The dominance of the

${ }^{5}$ For $\Lambda=2.5 \mathrm{fm}^{-1}$, we use the (b) fit of Ref. [21], since this lies smoothly between the $\Lambda=1.9 \mathrm{fm}^{-1}$ and $\Lambda=3.0 \mathrm{fm}^{-1}$ fits. 


\begin{tabular}{|c|c|c|c|c|c|c|c|c|c|c|c|}
\hline \multirow[b]{2}{*}{$k_{\mathrm{F}}$} & \multirow[b]{2}{*}{$\Lambda$} & \multicolumn{5}{|c|}{ Hartree-Fock } & \multicolumn{5}{|c|}{ Hartree-Fock + dominant second order } \\
\hline & & $T$ & $V_{\text {low } k}$ & $V_{c}$ & $V_{D}$ & $V_{E}$ & $T$ & $V_{\text {low } k}$ & $V_{c}$ & $V_{D}$ & $V_{E}$ \\
\hline \multirow[t]{4}{*}{1.0} & 1.6 & 12.44 & -19.62 & 1.65 & 0.42 & -0.22 & 15.50 & -26.58 & 1.49 & 0.34 & -0.29 \\
\hline & 1.9 & 12.44 & -18.18 & 1.67 & -0.25 & 0.40 & 16.29 & -26.81 & 0.85 & -0.09 & 0.55 \\
\hline & 2.1 & 12.44 & -17.35 & 1.67 & -0.42 & 0.62 & 16.92 & -27.04 & 0.11 & 0.05 & 0.79 \\
\hline & 2.3 & 12.44 & -16.56 & 1.67 & -0.56 & 0.81 & 17.60 & -27.27 & -0.89 & 0.43 & 0.85 \\
\hline \multirow[t]{4}{*}{1.2} & 1.6 & 17.92 & -31.47 & 5.37 & 1.31 & -0.64 & 20.86 & -37.66 & 4.59 & 1.03 & -0.65 \\
\hline & 1.9 & 17.92 & -28.95 & 5.61 & -0.81 & 1.18 & 21.80 & -37.38 & 3.99 & -0.50 & 1.28 \\
\hline & 2.1 & 17.92 & -27.51 & 5.67 & -1.37 & 1.84 & 22.87 & -37.53 & 2.27 & -0.37 & 1.82 \\
\hline & 2.3 & 17.92 & -26.13 & 5.70 & -1.86 & 2.42 & 24.32 & -37.95 & -0.38 & 0.51 & 1.78 \\
\hline \multirow[t]{4}{*}{1.35} & 1.6 & 22.67 & -42.47 & 10.75 & 2.59 & -1.21 & 26.09 & -47.85 & 8.73 & 1.96 & -1.12 \\
\hline & 1.9 & 22.67 & -38.82 & 11.95 & -1.69 & 2.34 & 26.75 & -46.72 & 9.14 & -1.16 & 2.24 \\
\hline & 2.1 & 22.67 & -36.74 & 12.19 & -2.91 & 3.68 & 28.05 & -46.47 & 6.99 & -1.33 & 3.22 \\
\hline & 2.3 & 22.67 & -34.77 & 12.30 & -3.97 & 4.89 & 30.06 & -46.45 & 3.10 & -0.35 & 3.26 \\
\hline
\end{tabular}

Table 2

Expectation values of the kinetic energy $(T), V_{\text {low } k}$ and the different $V_{3 \mathrm{~N}}$ contributions in $\mathrm{MeV}$. The expectation values are obtained with the Feynman-Hellman method, and $\Lambda$ and $k_{\mathrm{F}}$ are given in $\mathrm{fm}^{-1}$.

single-exchange terms holds for other densities and cutoffs as well.

Next, we compute the dominant second-order contributions to the energy per particle. The approximate second-order calculation is carried out in two steps. First, we convert the $3 \mathrm{~N}$ force into a density-dependent $\mathrm{NN}$ interaction $\bar{V}_{3 \mathrm{~N}}$ by summing the third particle over occupied states in the Fermi sea,

$$
\left\langle 12\left|\bar{V}_{3 \mathrm{~N}}\right| 1^{\prime} 2^{\prime}\right\rangle=\operatorname{Tr}_{\sigma_{3}, \tau_{3}} \int \frac{d \mathbf{k}_{3}}{(2 \pi)^{3}} n_{k_{3}} f_{\mathrm{R}}(123) f_{\mathrm{R}}\left(1^{\prime} 2^{\prime} 3\right)\left\langle 123\left|V_{3 \mathrm{~N}}\right| 1^{\prime} 2^{\prime} 3\right\rangle .
$$

A first-order calculation of $E^{(1)}$ using $\bar{V}_{3 \mathrm{~N}}$ includes the single-exchange contributions of the Hartree-Fock calculation with $V_{3 \mathrm{~N}}$. We then calculate the second-order contributions $E^{(2)}$ from $V_{\text {low } k}$ plus density-dependent $\bar{V}_{3 \mathrm{~N}}$,

$$
\begin{aligned}
\frac{E^{(2)}}{V} & =-\frac{1}{4} \prod_{i=1}^{4}\left(\operatorname{Tr}_{\sigma_{i}, \tau_{i}} \int \frac{d \mathbf{k}_{i}}{(2 \pi)^{3}}\right) n_{k_{1}} n_{k_{2}}\left(1-n_{k_{3}}\right)\left(1-n_{k_{4}}\right) \\
& \times \frac{\left|\left\langle 12\left|\left(V_{\text {low } k}+\bar{V}_{3 \mathrm{~N}}\right)\left(1-P_{12}\right)\right| 34\right\rangle\right|^{2}}{\epsilon_{k_{3}}+\epsilon_{k_{4}}-\epsilon_{k_{1}}-\epsilon_{k_{2}}}(2 \pi)^{3} \delta^{(3)}\left(\mathbf{k}_{1}+\mathbf{k}_{2}-\mathbf{k}_{3}-\mathbf{k}_{4}\right)
\end{aligned}
$$

For the intermediate-state integrations, the phase-space is angle-averaged and we use a continuous spectrum for $\epsilon_{k}=k^{2} /\left(2 m^{*}\right)$. The angle-averaging approximation is expected to be reliable [34]. Here the effective mass is determined 


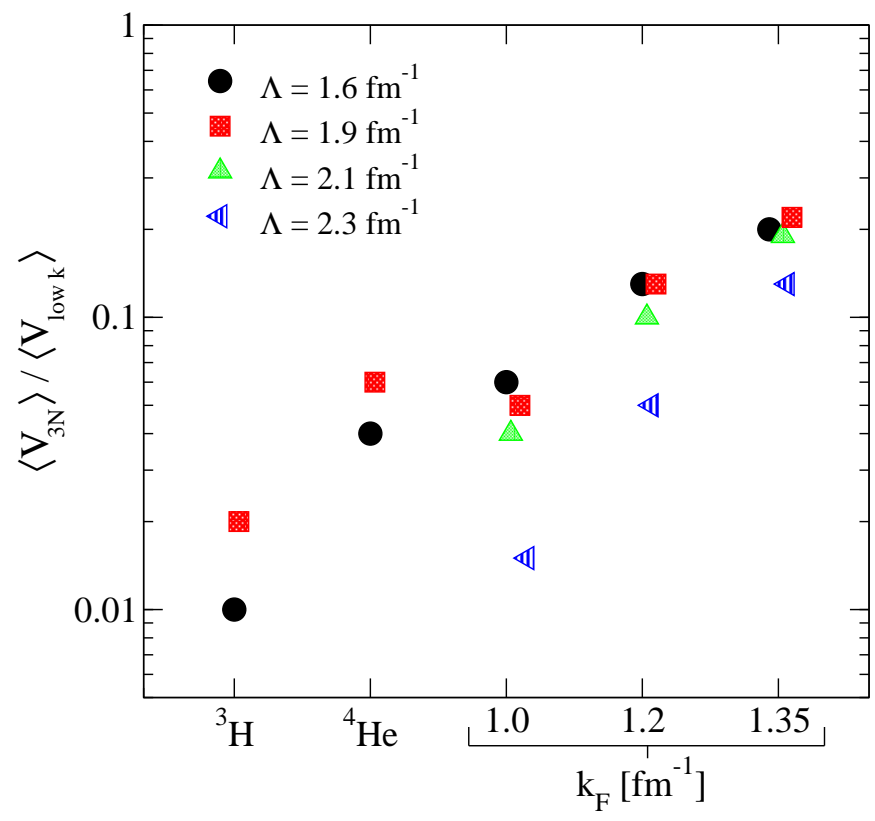

Fig. 7. Ratio of $V_{3 \mathrm{~N}}$ to $V_{\text {low } k}$ expectation values in the approximate second-order calculation. The expectation values are obtained by the Feynman-Hellman method. For nuclear matter we take for $\left\langle V_{3 \mathrm{~N}}\right\rangle$ the total $3 \mathrm{~N}$ contribution, whereas the values shown for ${ }^{3} \mathrm{H}$ and ${ }^{4} \mathrm{He}$ are the largest $3 \mathrm{~N}$ contributions (because of cancellations in the light nuclei).

from the first-order self-energy correction from $V_{\text {low } k}+\bar{V}_{3 \mathrm{~N}}$ at the Fermi surface,

$$
\frac{m^{*}}{m}=\left(1-\left.\frac{k}{m} \frac{\partial \Sigma(k)}{\partial k}\right|_{k=k_{\mathrm{F}}}\right)^{-1}
$$

where the spin-isospin independent part of the self-energy $\Sigma(k)$ is given by

$$
\Sigma(k)=\frac{1}{4} \operatorname{Tr}_{\sigma_{1}, \tau_{1}} \operatorname{Tr}_{\sigma_{2}, \tau_{2}} \int \frac{d \mathbf{k}_{2}}{(2 \pi)^{3}} n_{k_{2}}\left\langle 12\left|\left(V_{\text {low } k}+\bar{V}_{3 \mathrm{~N}}\right)\left(1-P_{12}\right)\right| 12\right\rangle .
$$

In this approximation, we find for the effective mass $m^{*} / m=0.72,0.67$ and 0.65 for $\Lambda=1.9 \mathrm{fm}^{-1}$ at $k_{\mathrm{F}}=1.0 \mathrm{fm}^{-1}, 1.2 \mathrm{fm}^{-1}$ and $1.35 \mathrm{fm}^{-1}$ respectively.

Our nuclear matter results including these dominant second-order contributions are shown in the right panel of Fig. 6 and are promising in a number of aspects. First, we observe that the second-order corrections move the saturation curves towards the empirical value. With the additional attraction, we find a minimum of $E / A \approx-(11-12) \mathrm{MeV}$ for Fermi momenta $k_{\mathrm{F}} \approx(1.2-1.35) \mathrm{fm}^{-1}$ over the cutoff range considered. Third-order particle-particle and hole-hole contributions are found to be small $(\lesssim 1 \mathrm{MeV})$. Second, the cutoff dependence is dramatically reduced when second-order contributions are included. We note that the determination of the $c_{i}$ are not unique $[10,33,35,36]$. For example, the values $c_{3}=-3.2 \mathrm{GeV}^{-1}$ and $c_{4}=5.4 \mathrm{GeV}^{-1}$ determined by Entem and Machleidt [10] would yield an additional $\sim 1-2 \mathrm{MeV}$ binding. We also 
emphasize that our calculation neglects second-order double-exchange contributions, which are expected to be attractive and nearly cutoff-independent. Furthermore, the effective mass approximation needs to be improved to treat the full momentum dependence of the Hartree-Fock self-energy.

Our results establish that the $3 \mathrm{~N}$ force drives saturation for low-momentum interactions. However, this does not imply that the $3 \mathrm{~N}$ contributions are unnaturally large. To provide further insight, we list in Table 2 the expectation values for the kinetic and potential energies in symmetric nuclear matter. The expectation values are obtained by the Feynman-Hellman method, where one multiplies the operator of interest in the Hamiltonian by a parameter $g$, so that $H^{g}=(H-O)+g O$, and computes $\langle O\rangle=\left.\left(d\left\langle H^{g}\right\rangle / d g\right)\right|_{g=1}$. The cutoff dependence of the expectation value for the kinetic energy $\langle T\rangle$ reflects the fact that correlations in the wave functions are not observable and are reduced as the cutoff is lowered. Note that $\langle T\rangle$ is significantly larger for large-cutoff interactions, see e.g., [8].

We also show in Fig. 7 the ratio of $\left\langle V_{3 \mathrm{~N}}\right\rangle /\left\langle V_{\text {low } k}\right\rangle$ for the triton, alpha particle, and nuclear matter at various densities. According to chiral power-counting estimates, a natural $3 \mathrm{~N}$ contribution should scale as $\left\langle V_{3 \mathrm{~N}}\right\rangle \sim(Q / \Lambda)^{3}\left\langle V_{\mathrm{NN}}\right\rangle$, where $Q$ is a typical momentum scale of the low-energy system. Our results for the expectation values show that the $3 \mathrm{~N}$ contributions are not unnatural; for example, the ratio $\left\langle V_{3 N}\right\rangle /\left\langle V_{\text {low } k}\right\rangle\left(\Lambda / k_{\mathrm{F}}\right)^{3}$ is $0.31,0.51,0.53$, and 0.35 for the second-order calculation at $k_{\mathrm{F}}=1.2 \mathrm{fm}^{-1}$ and $\Lambda=1.6 \mathrm{fm}^{-1}, 1.9 \mathrm{fm}^{-1}$, $2.1 \mathrm{fm}^{-1}$, and $2.3 \mathrm{fm}^{-1}$ respectively. Nevertheless, the role of $3 \mathrm{~N}$ forces in driving saturation means that we will ultimately want to identify a power counting for finite density that includes both $\mathrm{NN}$ and $3 \mathrm{~N}$ Hartree-Fock contributions at leading order. It is also interesting to note that the deviation from the empirical saturation point is compatible with a $3 \mathrm{~N}$ or $4 \mathrm{~N}$ force contribution of order $(Q / \Lambda)^{4}$.

Finally, as was previously found for the binding energies of $A=3,4 \mathrm{nu}$ clei [21], we find that the $3 \mathrm{~N}$ force may also be perturbative in nuclear matter calculations for cutoffs $\Lambda \lesssim 2 \mathrm{fm}^{-1}$. For example, the expectation value of $\left\langle V_{\text {low } k}\right\rangle$ for $k_{\mathrm{F}}=1.2 \mathrm{fm}^{-1}$ in the absence of $3 \mathrm{~N}$ forces are $\left\langle V_{\text {low } k}\right\rangle=-37.5 \mathrm{MeV}$, $-37.9 \mathrm{MeV},-38.2 \mathrm{MeV}$, and $-38.5 \mathrm{MeV}$ for $\Lambda=1.6 \mathrm{fm}^{-1}, 1.9 \mathrm{fm}^{-1}, 2.1 \mathrm{fm}^{-1}$, and $2.3 \mathrm{fm}^{-1}$ respectively. Comparing these with the expectation values in Table 2 , we find that $3 \mathrm{~N}$ effects in the wave functions are small. In contrast, the expectation value of the Argonne $v_{18}$ potential changes by $1.6 \mathrm{MeV}$ after resummations when the Urbana IX $3 \mathrm{~N}$ interaction is included [8]. 


\section{Conclusions and Summary}

In this paper, the nonperturbative nature of inter-nucleon interactions is explored by varying the momentum cutoff of a two-nucleon potential. Conventional force models, which have large cutoffs, are nonperturbative because of strong short-range repulsion, the iterated tensor interaction, and the presence of bound or nearly-bound states. But for low-momentum interactions with cutoffs around $2 \mathrm{fm}^{-1}$, the softened potential combined with Pauli blocking leads to corrections in nuclear matter in the particle-particle channel that are well converged at second order in the potential, suggesting that perturbation theory (as in a loop expansion) can be used in place of Brueckner resummations. A Weinberg eigenvalue analysis provides quantitative backing to these observations.

We present the first calculations of nuclear matter using the low-momentum two-nucleon $V_{\text {low } k}$ with a corresponding $3 \mathrm{~N}$ force from chiral EFT, which are fit in free space and to binding energies of three-nucleon systems with no adjustments when applied at finite density. This combination exhibits nuclear binding in the Hartree-Fock approximation and becomes significantly less cutoff dependent with the inclusion of the dominant second-order contributions. The role of the $3 \mathrm{~N}$ force is essential to obtain saturation, but the contribution to the total potential energy is still compatible with EFT power-counting estimates.

At lower cutoffs around $2 \mathrm{fm}^{-1}$, the iterated tensor interaction in the twobody sector does not play a major role in nuclear saturation, in contrast to the conventional wisdom. We emphasize, however, that the relative importance of contributions to observables from the tensor force or from three-body forces are scale or resolution dependent. Renormalizing the potential to a different cutoff redistributes these contributions. Our point is that potentials with low-momentum cutoffs may be superior for practical nuclear many-body calculations.

We further emphasize that the use of $V_{\text {low } k}$ is not a replacement for EFT but can be used to improve EFT interactions for many-body applications. The strategy is to start with a consistently truncated chiral EFT (including many-nucleon interactions), using the highest feasible cutoff $\Lambda$ to minimize the truncation error in matching. Then we evolve the EFT to lower cutoffs (lower resolution) using the RG. This running does not, in the present case, involve large separations of scale that would justify a truncated derivative expansion, so we keep induced operators to all orders to prevent the truncation error from growing.

One could be misled into thinking a higher cutoff implies that the physics is 
more valid. As stressed by Lepage, one should match a low-energy theory to data or an underlying theory with a cutoff close to the scale at which unknown physics starts to be resolved [9]. There is no advantage to putting it higher, as it can be counterproductive because counterterms (which translates into the potential here) must cancel loop contributions from intermediate states that are incorrectly represented. ${ }^{6}$ But this does not mean that EFT calculations cut off at the breakdown scale are optimal for applications to nuclei. Instead, one should choose a resolution appropriate to the problem by matching the chiral EFT at the breakdown scale $\left(\Lambda \approx 3-4 \mathrm{fm}^{-1}\right)$ and then evolve downward. We find that the decrease in resolution to $\Lambda \sim 2 \mathrm{fm}^{-1}$ is particularly important for reducing the tensor force.

The application of chiral EFT in perturbation theory by Lutz et al. [37] and Kaiser et al. [38] may be justified in part by our results, although further detailed comparisons are needed. We expect, however, that the convergence will be improved for smaller cutoffs $\left(\Lambda=3.28 \mathrm{fm}^{-1}\right.$ in [38]). In contrast to our results, which are predictions based on potential fits to scattering observables with relatively small residual cutoff dependence of observables, Kaiser et al. fine tune the cutoff to simulate the effects of omitted contact terms. Contact interactions are included in $V_{\text {low } k}$ and their contributions to nuclear matter are important. In addition, $2 \pi$-exchange $3 \mathrm{~N}$ contributions, which are essential for saturation in the present approach, are not included in Ref. [38]. It is conceivable that a substantial part of our $3 \mathrm{~N}$ force contributions are captured by the explicit Delta isobars included in Fritsch et al. [39], since the low-energy constants $c_{3}$ and $c_{4}$ are to a large extent saturated by the Delta isobar [40]. Therefore, a detailed comparision with this work will be very interesting for the future.

The present calculations can be improved in several respects, which is reflected in residual cutoff dependence of the energy per particle in nuclear matter. First, the current calculations are not complete at second order in many-body perturbation theory. We expect that the missing second-order contributions are, in fact, more important than omitted higher-order contributions. The double-exchange $c$-terms are expected to give attractive and nearly cutoffindependent contributions. Thus, a full second-order calculation is a high priority. Other improvements would be a better treatment of self-consistency at second order. In parallel, we will use the cutoff dependence of $V_{\text {low } k}$ as a tool to develop a power counting at finite density that specifies the appropriate truncations and justifies our conclusions.

With the present three-body force, a better determination of the $c_{i}$ coefficients

6 In other words, the model-dependent cores in the conventional NN potentials mandate model-dependent parts in the $3 \mathrm{~N}$ force to counteract the effects of the former. 
is needed, since $c_{3}$ and $c_{4}$ contribute at the $\mathrm{MeV}$ level. Of course, it will be important to eventually evolve the many-body forces to lower cutoffs at the same time as the two-nucleon force is renormalized, as this will generate better estimates of higher-order three-body and four-body contributions. In addition, the impact of using cutoffs for $\Lambda<2 k_{\mathrm{F}}$ needs further investigation.

Finally, there are particle-hole contributions to consider. These contribute to the energy at third order. While we have shown that nuclear matter is perturbative in the particle-particle channel and that $3 \mathrm{~N}$ forces naturally provides a saturation mechanism, there is still the possibility that a nonperturbative summation in the particle-hole channels is required [41]. The lore with conventional potentials is that these correlations are important for the nuclear response but not for the energy of bulk nuclear matter. If this also holds for low-momentum potentials, then we can conclude that nuclear matter under ordinary conditions can be treated perturbatively in the inter-nucleon forces.

For applications to astrophysics, however, we may seek higher densities (e.g., for applications to the neutron star equation of state). The increase in threebody contributions with density above saturation implies that one should only do controlled extrapolations to higher density. Controlled extrapolations are also needed to reliably describe neutron- or proton-rich nuclei towards the drip lines. The use of a variable cutoff and natural estimates of omitted contributions is the ideal tool for such investigations.

\section{Acknowledgements}

We are grateful to Norbert Kaiser for pointing out the $c_{4}$ Fock contribution. We thank Gerry Brown, Bengt Friman, Chuck Horowitz, Brian Serot and Bira van Kolck for useful comments and discussions. This work was supported in part by the National Science Foundation under Grants No. PHY-0098645, No. PHY-0244822, and No. PHY-0354916, and by the US Department of Energy under Grants No. DE-FG02-87ER40365, DE-FC02-01ER41187 and DE-FG02-00ER41132.

\section{A Pionic three-nucleon force contributions without regulator}

In this Appendix, we provide analytical expressions for the $1 \pi$ - and $2 \pi$-exchange $3 \mathrm{~N}$ force contributions at the Hartree-Fock level, when the regulator $f_{\mathrm{R}}$ is neglected. Analogous $2 \pi$-exchange contributions with explicit Delta isobars have been evaluated in [39]. This provides an estimate for these $3 \mathrm{~N}$ contributions and a check for the numerical integration with regulator. 
For the $1 \pi$-exchange $3 \mathrm{~N}$ interaction $\left(V_{D}\right)$, we obtain from Eq. $(23)$ with $f_{\mathrm{R}}=1$

$$
\begin{aligned}
\frac{E_{D}^{(1)}}{A} & =\frac{g_{A} c_{D}}{f_{\pi}^{4} \Lambda_{\chi}} \frac{3}{288(2 \pi)^{4}}\left[32 k_{\mathrm{F}}^{6}-72 k_{\mathrm{F}}^{4} m_{\pi}^{2}+12 k_{\mathrm{F}}^{2} m_{\pi}^{4}\right. \\
& \left.+96 k_{\mathrm{F}}^{3} m_{\pi}^{3} \arctan \left(\frac{2 k_{\mathrm{F}}}{m_{\pi}}\right)-\left(36 k_{\mathrm{F}}^{2} m_{\pi}^{4}+3 m_{\pi}^{6}\right) \log \left(1+\frac{4 k_{\mathrm{F}}^{2}}{m_{\pi}^{2}}\right)\right]
\end{aligned}
$$

and for the $2 \pi$-exchange $3 \mathrm{~N}$ interaction $\left(V_{c}\right)$, Eq. (26) leads to

$$
\begin{aligned}
\frac{E_{c}^{(1)}}{A} & =\frac{g_{A}^{2} m_{\pi}^{2} c_{1}}{k_{\mathrm{F}}^{3} f_{\pi}^{4}} \frac{9}{2(2 \pi)^{4}}\left[\frac{k_{\mathrm{F}}^{3}}{3 m_{\pi}} \partial_{m_{\pi}}\left(m_{\pi}^{6} \int_{0}^{u} x d x G_{s}(x, u)\right)\right. \\
& \left.+\frac{m_{\pi}^{7}}{128} \int_{0}^{u} x^{2} d x G_{v}^{2}(x, u)\right] \\
& +\frac{g_{A}^{2} c_{3}}{k_{\mathrm{F}}^{3} f_{\pi}^{4}} \frac{9}{4(2 \pi)^{4}}\left[\frac{m_{\pi}^{9}}{72} \int_{0}^{u} d x\left(3 G_{s}^{2}(x, u)+G_{t}^{2}(x, u)\right)\right. \\
& -\frac{2 k_{\mathrm{F}}^{3} m_{\pi}^{6}}{3} \int_{0}^{u} x d x G_{s}(x, u)-\frac{k_{\mathrm{F}}^{3} m_{\pi}}{3} \partial_{m_{\pi}}\left(m_{\pi}^{6} \int_{0}^{u} x d x G_{s}(x, u)\right) \\
& \left.+\frac{g_{A}^{2} m_{\pi}^{9} c_{4}}{k_{\mathrm{F}}^{3} f_{\pi}^{4}} \frac{3}{(4 \pi)^{4}} \int_{0}^{u} d x\left(G_{t}^{2}(x, u)-G_{s}^{2}(x, u)\right)\right]
\end{aligned}
$$

Here $u=k_{\mathrm{F}} / m_{\pi}$ and the auxiliary functions $G_{s}(x, u), G_{v}(x, u)$ and $G_{t}(x, u)$ are defined as

$$
\begin{aligned}
G_{s}(x, u) & =\frac{4 u x}{3}\left(2 u^{2}-3\right)+4 x(\arctan (u+x)+\arctan (u-x)) \\
& +\left(x^{2}-u^{2}-1\right) \log \frac{1+(u+x)^{2}}{1+(u-x)^{2}} \\
G_{v}(x, u) & =\frac{1}{x^{2}}\left(\left(u^{4}-2 u^{2}\left(x^{2}-1\right)+\left(x^{2}+1\right)^{2}\right) \log \frac{1+(u+x)^{2}}{1+(u-x)^{2}}\right. \\
& \left.-4 u x\left(x^{2}+u^{2}+1\right)\right) \\
G_{t}(x, u) & =\frac{u x}{6}\left(8 u^{2}+3 x^{2}\right)-\frac{u}{2 x}\left(1+u^{2}\right)^{2} \\
& +\frac{1}{8}\left(\frac{\left(1+u^{2}\right)^{3}}{x^{2}}-x^{4}+\left(1-3 u^{2}\right)\left(1+u^{2}-x^{2}\right)\right) \log \frac{1+(u+x)^{2}}{1+(u-x)^{2}} .
\end{aligned}
$$




\section{References}

[1] H.A. Bethe, Ann. Rev. Nucl. Sci. 21 (1971) 93.

[2] A. D. Jackson, Ann. Rev. Nucl. Part. Sci. 33 (1983) 105; R. Machleidt, Adv. Nucl. Phys. 19 (1989) 189; H. Müther and A. Polls, Prog. Part. Nucl. Phys. 45 (2000) 243.

[3] S.R. Beane, P.F. Bedaque, M.J. Savage and U. van Kolck, Nucl. Phys. A700 (2002) 377.

[4] S. Fleming, T. Mehen and I.W. Stewart, Nucl. Phys. A677 (2000) 313.

[5] R.B. Wiringa, V.G.J. Stoks and R. Schiavilla, Phys. Rev. C51 (1995) 38.

[6] S.C. Pieper and R.B. Wiringa, Ann. Rev. Nucl. Part. Sci. 51 (2001) 53.

[7] S.C. Pieper, R.B. Wiringa and J. Carlson, Phys. Rev. C70 (2004) 054325.

[8] A. Akmal, V.R. Pandharipande and D.G. Ravenhall, Phys. Rev. C58 (1998) 1804 .

[9] G.P. Lepage, "How to Renormalize the Schrödinger Equation", Lectures given at 9th Jorge Andre Swieca Summer School: Particles and Fields, Sao Paulo, Brazil, February, 1997, nucl-th/9706029.

[10] D.R. Entem and R. Machleidt, Phys. Rev. C68 (2003) 041001(R).

[11] E. Epelbaum, W. Glöckle and U.G. Meißner, Nucl. Phys. A747 (2005) 362.

[12] S.K. Bogner, T.T.S. Kuo, A. Schwenk, D.R. Entem and R. Machleidt, Phys. Lett. B576 (2003) 265.

[13] S.K. Bogner, T.T.S. Kuo and A. Schwenk, Phys. Rept. 386 (2003) 1.

[14] S.K. Bogner, A. Schwenk, T.T.S. Kuo and G.E. Brown, nucl-th/0111042.

[15] E. Epelbaoum, W. Glöckle, A. Krüger and U.G. Meißner, Nucl. Phys. A645 (1999) 413.

[16] F. Coester, F. Cohen, B. Day and C.M. Vincent, Phys. Rev. C1 (1970) 769; M.I. Haftel and F. Tabakin, Phys. Rev. C3 (1971) 921.

[17] J. Kuckei, F. Montani, H. Müther and A. Sedrakian, Nucl. Phys. A723 (2003) 32.

[18] S. Fujii, E. Epelbaum, H. Kamada, R. Okamoto, K. Suzuki and W. Glöckle, Phys. Rev. C70 (2004) 024003.

[19] A. Nogga, H. Kamada and W. Glöckle, Phys. Rev. Lett. 85 (2000) 944.

[20] P. Navratil and W.E. Ormand, Phys. Rev. C68 (2003) 034305.

[21] A. Nogga, S.K. Bogner and A. Schwenk, Phys. Rev. C70 (2004) 061002(R). 
[22] G. Röpke, L. Münchow and H. Schulz, Nucl. Phys. A379 (1982) 536.

[23] B.L. Friman, J. Niskanen and E.M. Nyman, Nucl. Phys. A383 (1982) 285.

[24] S. Weinberg, Phys. Lett. B251 (1990) 288.

[25] M. Baldo, Ø. Elgarøy, L. Engvik, M. Hjorth-Jensen and H.-J. Schulze, Phys. Rev. C58 (1998) 1921.

[26] S. Weinberg, Phys. Rev. 131 (1963) 440.

[27] G.E. Brown, Unified Theory of Nuclear Models and Forces, North-Holland, Amsterdam, Third Edition, 1971.

[28] M. Hjorth-Jensen, T.T.S. Kuo and E. Osnes, Phys. Rept. 261 (1995) 125.

[29] M. Baldo, in Nuclear Methods and the Nuclear Equation of State, International Review of Nuclear Physics, Vol. 8, World Scientific, 1999.

[30] A. Lejeune, U. Lombardo and W. Zuo, Phys. Lett. B477 (2000) 45.

[31] U. van Kolck, Phys. Rev. C49 (1999) 2932.

[32] E. Epelbaum, A. Nogga, W. Glöckle, H. Kamada, U.G. Meißner and H. Witala, Phys. Rev. C66 (2002) 064001.

[33] M.C.M. Rentmeester, R.G.E. Timmermans and J.J. de Swart, Phys. Rev. C67 (2003) 044001.

[34] K. Suzuki, R. Okamoto, M. Kohuo and S. Nagata, Nucl. Phys. A665 (2000) 92 .

[35] N. Fettes, U.-G. Meißner and S. Steininger, Nucl. Phys. A640 (1998) 199.

[36] P. Büttiker and U.-G. Meißner, Nucl. Phys. A668 (2000) 97.

[37] M. Lutz, B. Friman and C. Appel, Phys. Lett. B474 (2000) 7.

[38] N. Kaiser, S. Fritsch and W. Weise, Nucl. Phys. A697 (2002) 255.

[39] S. Fritsch, N. Kaiser and W. Weise, Nucl. Phys. A750 (2005) 259.

[40] V. Bernard, N. Kaiser and U.-G. Meißner, Nucl. Phys. A615 (1987) 483.

[41] T. Wettig and A.D. Jackson, Phys. Rept. 237 (1994) 325. 